\title{
Towards an Evolutionary Account of Conceptual Change in Mathematics: Proofs and Refutations and the Axiomatic Variation of Concepts
}

\author{
Thomas Mormann
}

1. Introduction. Although Lakatos's Proofs and Refutations (henceforth (PR)) is one of the classics of philosophy of mathematics in the twentieth century few attempts have been made to build on its ideas. ${ }^{1}$ Rather, quite often (PR) has become the object of respectful reference and detailed exegetical efforts. Of course, Lakatos is credited for having taught philosophers that philosophy of mathematics has to take into account the history and practice of mathematics. This orientation towards the history and practice of the discipline is certainly a definite progress over the ahistorical traditions of logicism, formalism, platonism, and intuitionism. It should help to get rid of the most pernicious vice philosophy of mathematics is plagued with, to wit, elementarism which considers elementary arithmetics of natural numbers or Euclidean geometry as typical for all of mathematics. I think, however, there is more to learn from Lakatos than just a general orientation towards history and practice of real-life mathematics. As I want to show in this paper, these insights concern the role of inventing and varying mathematical concepts. More precisely, I want to use Lakatos's ideas of "concept-formation" and "conceptstretching" to sketch an evolutionary theory of mathematical knowledge which takes axiomatic variation of concepts as the fundamental driving force of the ongoing evolution of mathematics (PR, 83ff). ${ }^{2}$

Although Lakatos considers concept-stretching only for informal, not axiomaticed mathematics, I contend that it is not restricted to this realm. Rather,

\footnotetext{
${ }^{1}$ There are, of course, exceptions. For instance, Fine (1981) attempts to embed Lakatos's account of concept-formation and concept-stretching as pursued in (PR) in a more general theory of concept-refinement not restricted to mathematical concepts only but embracing the conceptual development of all kinds of scientific concepts. As it seems, his sketchy outline has not been taken up by other authors.
}

2 Of course, an evolutionary theory of (scientific) concepts is not new at all. For instance, Toulmin offers an evolutionary description of the historical development of theories, and, more generally, intellectual disciplines, in the framework of a population model (Toulmin 1972, Part II). Toulmin's account, however, is deliberately general. He is interested in the general mechanisms of the evolution of ideas, not in the specific mechanisms of a special discipline such as mathematics. The point I want to make in the following is that the mechanism of axiomatic concept variation is characteristic for the development of mathematical concepts and deserves a closer study than has hitherto carried out. 
the role of conceptual variation (which I take to be a "neutral" term for Lakatosian concept-formation, concept-stretching and related conceptual activities) becomes even more important for axiomatic mathematics.

In the following I propose to generalize Lakatos's approach of conceptstretching in such a way that history and practice of modern axiomatic mathematics can be conceived of as an evolution of mathematical concepts based on axiomatic concept variation. In other words, I contend that a philosophical understanding of mathematics may be provided by conceiving it as a conceptual evolution. This implies that, in some sense, mathematical concepts are to be considered as more basic than mathematical theorems. ${ }^{3}$ At first look, this contention may be considered as absurd. According to a time-honored piece of philosophical wisdom mathematical knowledge is deductive knowledge deposited in proven theorems. We know what is a deductive proof and a theorem, at least much better than what is to be understood by a mathematical concept. Thus, it may appear unplausible, to say the least, to base a philosophical understanding of mathematical knowledge on the elusive notion of a mathematical concept. Hence, I hasten to add that the concept-based approach outlined in this paper does not dismiss the notions of theorem and proof at all. Rather, they provide the necessary environment for mathematical concepts which never occur in isolation but always in the context of mathematical theorems. The importance of concepts depends on the importance of theorems in which they occur.

Mathematical concepts may be conceived of as entities on which one may conduct trials or experiments. The trials or experiments are the attempts of proving theorems in which the concept in question occupies a central position. Then, a successful experiment, i.e., a valid proof, may be considered as an outcome positive for the concept in question. For instance, to take Lakatos's example, the "Euler-experiment" on some variant of the polyhedron concept is the attempt to prove a version of the Euler conjecture for it. Usually, several conceptual variants compete in such an experiment. If several attempts of proving a conjecture fail for a conceptual variant, or only lead to an uninteresting contrived version of the original conjecture, that variant may be considered as a failure. In this way, conjectures provide objective challenges or

\footnotetext{
${ }^{3}$ The key-role of concepts for the development of mathematical knowledge is clearly acknowledged in (PR): As Lakatos asserts in a footnote: "The key to Euler's result (i.e., the Euler conjecture, T.M.) was just the invention of the concepts of vertex and edge ..." In contrast, Descartes did not found the key for the formulation of Euler's conjecture, since he "did not make a conscious revolutionary change to the concepts of 0 -dimensional vertices, 1-dimensional egdes and 2dimensional faces as a necessary and sufficient basis for the full characterization of polyhedra." (PR, p.6, footnote 1)
} 
survival tasks for a group of competing variants of concepts which have to cope with them. This does not mean, however, that there is a one-way relation between concepts and theorems in the sense that concepts have to strictly respond to the rules defined by the theorems. Often, new conceptual variants give rise to new theorems which cannot be proved or even formulated, for the older conceptual variants. These new theorems alter the "theorematical" environment and change the terms of competition for future conceptual variants. Thereby, even the assessment of past conceptual achievements may change. The main thesis of this paper claims that this competition of conceptual variants may be described in the framework of an evolutionary theory which conceptualizes the evolution of mathematical knowledge as a selection process of conceptual variants taking place in a "world" whose challenges are determinded by varying theorematical environments.

The outline of this paper is as follows: in section 2 we take as our point of departure Lakatos's notion of concept-stretching. It may be considered as an informal precursor of the more powerful notion of axiomatic concept variation which is characteristic for the conceptual practice of modern mathematics. As an early example of an axiomatic concept variation we consider Hamilton's construction of the quaternions. In section 3 the basic principles of an elementary theory of evolution are proposed. In section 4 these principles are applied to the evolution of mathematical concepts. As an example of an evolutionary account, a sketch of the evolution of some conceptual variants of the integral is offered in section 5 . In section 6 we conclude with some remarks on the specific features of the evolution of mathematical concepts which need further investigation.

2. Conceptual Change: Concept-stretching and Axiomatic Variation. Traditionally, mathematical concepts have been conceived of as citizens of some Platonic realm. This conception rendered them immutable and eternal entities. Lakatos was one of the first philosophers who did not conceive of mathematical concepts in that manner. For him, mathematical concepts might undergo substantial alterations and changes, to wit, concept-stretching, or the reversal, concept-contracting. This is to say that mathematical concepts do not exhibit the immutability and eternity platonistic accounts use to ascribe to them; mathematical concepts vary. Lakatos develops a sort of dialectic of proof and counterexamples which intends to shed some light on the process of the variation of concepts. All this is has been the subject of detailed discussion and interpretation (cf. Koetsier 1991, Larvor 1997), and needs no rehearsal. It 
is sufficient to note that a large part of mathematical practice may be described as an activity dealing with mathematical concepts, be it conceptstretching in the sense of Lakatos, or generalizing, specializing, adapting, or what sort of varying concepts whatsoever. As a result of this complex concepttrafficking, typical mathematical concepts come along in a multiplicity of variants. For instance, Lakatos gives five or six different variants of the keyconcept "polyhedron" (cf. PR, pp. 14 - 22) which, according to his notorious footnotes, have actually occurred in the mathematical history of that concept. ${ }^{4}$ Lakatos restricts the practice of concept-stretching to the realm of informal, non-axiomatized mathematics. As soon as it comes to formal axiomatized mathematics, he assumes that concept-stretching comes to an end, which is, according to him, a reason why modern axiomatic mathematics lacks much of the freedom and the excitement of earlier times. I think this is quite wrong. Rather, as Corfield has emphasized:

"[t]he appropriate use of rigorous definition and axiomatization has not acted as a hobble on the creativity of mathematicians, but rather an invaluable tool in the forging of new mathematical theories and the extension of old ones." Corfield 1997, 100).

As Corfield points out, for established modern mathematical theories, the concepts involved are usually defined by systems of axioms. And it is precisely these systems of axioms which enable us to vary mathematical concepts in a very effective and conscious way not available for an informally formulated "intuitive" mathematics. Although I fully endorse Corfield's enthusiasm for the creative role of the axiomatic method I have reservations on his usage of the notion of "forging". When one is "forging" an object, e.g. an instrument or, metaphorically, a theory, one is assumed of having total control over the product one is forging. One knows what will be the result of one's doing. This, however, most often is not the case for mathematical concepts. The inventors of new mathematical concepts often don't know, what exactly will be the outcome and how their mind-children will score in the competition. Of course, they aren't completely in the dark. After all, they have designed the new concepts to do some definite useful work. But often they have modify to their original intentions and to reshape them in the presence of resistance and

\footnotetext{
${ }^{4}$ As we shall see in section 5 , this number is not at all an exaggeration. For instance, the number of variants of the concept of the integral can only be guessed. Even if we restrict counting to "modern" variants, one may distinguish more than one hundred from Cauchy onwards (cf. Hawkins 1970, Henstock 1991).
} 
obstacles they had not foreseen and are unable to overcome.

At first glance, such resistance in the conceptual realm of mathematics may look strange, since philosophers have repeatedly emphasized the freedom of mathematics: anything that is not inconsistent might be possible. Furthermore, the axiomatic method has been hailed for having fully brought to the fore this unique freedom of mathematics. A closer look on the practice of mathematics, however, reveals that the alleged freedom of mathematics is far from being a total one. Quite the contrary, the conceptual practice of mathematics is severely restricted by the rules we have to follow in our conceptual practices. As every mathematician knows, the conceptual practice of mathematics soon uses to become difficult. The workings of the mind may lead the mind itself into difficult and sometimes unsolvable problems. ${ }^{5}$ Thus, a primary task of philosophy of mathematics is to explain this somewhat paradoxical fact.

Recently, Pickering contended that this restricted freedom of conceptual practices such as mathematics may be conceived of as a special case of what he calls the "mangle of practice" in scientific practices in general (Pickering 1995). According to him, the essential ingredient for getting a non-distorted "non-abstract" picture of science (mathematics included) is to conceive this endeavour as a temporarily situated practice in which a human and a nonhuman agency interact. The contours of both agencies are not fixed from the outset, but emerge in the temporality of practice. That is to say, they are never to be considered as fully determined and complete. Seen from the perspective of the human part, this means that the goals and intentions of the scientist are continuously under revision. Scientists do not simply fix their goals ("prove the Euler's conjecture") once and for all and stick to them, come what may. Rather, they find themselves engaged in the "mangle of practice" in which their intentions undergo a permanent goal-revising dialectic of resistance and accommodation in such a way that the outcome only remotely resembles the original plannings (cf. Pickering 1995, 22-23). ${ }^{6}$

\footnotetext{
${ }^{5}$ This has turned out to be no coincidence. One may interpret the uncompleteness theorems as stating that the non-trivial workings of the mind necessarily lead to unsolvable problems.

${ }^{6}$ Pickering takes (PR) as a case of the mangle in conceptual practice: "The exhibition of novel counterexamples to specific proofs of the theorem counts, in my terminology, as the emergences of resistances, and Lakatos describes very nicely the revision of proof precedures as open-ended accommodation to such resistances, with interactive stabilization amounting to the reconciliation of such precedures to given counterexamples." Pickering (1995, 119, Footnote) To a large extent, this may be correct. But I think, Pickering overestimates the open-endedness of Lakatos's account. After all, even in the second chapter of (PR), in which he deals with Poincaré's proof of Euler's theorem, he concentrates on that theorem and nothing else. The reason may be he is still in the grip of a Popperian "statement view" of (mathematical and scientific) theories according to which single statements occupy the centre stage of scientific theorizing.
} 
As an example of the mangle of conceptual practice characteristic for the restricted freedom of the axiomatic variation of concepts let us consider the episode of Hamilton's invention of the quaternions (cf. Pickering 1995). Originally, Hamilton intended to generalize the geometric representation of complex numbers in the 2-dimensional Euclidean plane to get a threedimensional analogue:

“ $\sqrt{ }-1$ [being] perpendicular to 1 , it is natural to conceive that there may be another sort of $\sqrt{ }-1$, perpendicular to the plane itself. Let this new imaginary be $\mathrm{j}$; so that $\mathrm{j} 2=-1$, as well as $i 2=-1$. A point in space may suggest the triplet x + iy + jz." (Hamilton 1967, 103)

At this stage, Hamilton aimed at a rather straight-forward variation of complex numbers: instead of couples $(x, y)$ he considered the triples $(x, y, z)$, and attempted to generalize the arithmetics of complex numbers to their envisaged 3-dimensional counterpart.

The conceptual environment which this variant had to cope with was determined by the arithmetic laws of complex numbers which may be succinctly described by the following formulas:

$$
(x, y)+\left(x^{*}, y^{*}\right)=\left(x+x^{*}, y+y^{*}\right),(x, y) \bullet\left(x^{*}, y^{*}\right)=\left(x x^{*}-y y^{*}, x y^{*}+x^{*} y\right)
$$

That is to say, Hamilton was looking for 3-dimensional analogues of these laws. Of course, the extension of the additive laws (i.e., associativity, commutativity etc.) posed no problems. The multiplicative law turned out to be crucial test the envisaged triple numbers had to pass. ${ }^{7}$ He tried for more than fifteen years to find one, but never succeeded. Today, it is well-known that such a 3-dimensional extension of real numbers cannot exist. ${ }^{8}$ Rather, as Hamilton discovered in 1843 one must do a further step towards a 4-dimensional generalization of the real numbers leading to quadruplets $(x, y, z, w)$ as a viable variant of the complex numbers $(x, y)$. This variant of complex numbers (or, more remotely of real numbers) are called the hypercomplex numbers or quaternions $\mathrm{H}$. They do not satisfy commutative multiplication. Rather, if $1, i, j$ and $k$ denote the standard basis of $\mathrm{H}$ the multiplicative behaviour of quaternions is determined

\footnotetext{
7 This was clearly recognized by Hamilton. After he had found the 4-dimensional quaternions, he wrote: "But I considered it essential to try whether my equations were consistent with the law of moduli (i.e., the law of multiplication), ... without which consistence being verified, I should have regarded the whole speculation as a failure." (Hamilton 1967, 108)

${ }^{8}$ Already Hamilton could have proved this (cf. Ebbinghaus et al. 1983,131)
} 
by the following law:

$$
\mathrm{i} 2=\mathrm{j}^{2}=\mathrm{k} 2=\mathrm{i} \bullet \mathrm{j} \bullet \mathrm{k}=-1
$$

As is easily seen, (2.2) leads to an anti-commutative multiplication for $\mathrm{i}, \mathrm{j}$, and $k$, i.e., $i \bullet j=-j \bullet i=k$, etc. Thus, Hamilton's new generalized complex numbers $\mathrm{H}$, obtained by an axiomatic concept-variation concerning the multiplicative law do not satisfy the original arithmetic laws required for "honest" numbers. Hamilton invented a variant of complex numbers with rather different features than those he originally had looked for.

It should be noted that the axiomatic variation of concepts as it is exhibited in Hamilton's invention shows a feature which defies a strictly Lakatosian account, namely it does not leave intact the theorems or laws which originally were considered as the touchstones of its respectability. In (PR), the concepts of the polyhedron may be stretched or even teared, Euler's conjecture, however, never underwent any variation. ${ }^{9}$ As he started, Hamilton seems to have considered the arithmetic laws of the complex numbers in a similar vein as Lakatos understood the Euler Theorem, to wit, as a sort of experimentum crucis. It must be considered as a sign of Hamilton's genius that he was prepared to revise the touchstone laws. Thereby opening the gate for a "new world" whose riches he himself only dimly realized. In modern terms, this "new world" may be described as the theory of division algebras and related topics. In quite the same manner, most of the newly generated variants of mathematical concepts do not satisfy all theorems which hold for their ancestors. The dialectics of resistance and accommodations which steers the axiomatic variation of concepts is more flexible than Lakatos's Popperian account which is fixated on the original "bold conjecture".

Hamilton believed that the quaternions would open a new era for mathematics. This turned out to be an exaggeration. Modern mathematicians see the importance of Hamilton's quaternions not so much in themselves. After all, $\mathrm{H}$ has turned out to be a not too interesting variant of a division algebra. Rather, the importance of Hamilton's construction resides in the methodological fact that it may be conceived of as one of the first conscious and deliberate cases

\footnotetext{
${ }^{9}$ This claim contradicts Koetsier's reconstruction of Lakatos's argument. According to Koetsier, $[(P R)]$ "shows us a quite dramatic successions of versions of Euler's theorem for polyhedra, $V$ - E $+\mathrm{F}=2, \ldots$, each version being temporarily the best until it is refuted and has to be replaced by its successor." (Koetsier 1991, 24) All the "different" version of Euler's theorem have the following form: "For all X-polyhedra $V-E+F=2$." Here, "X-polyedra is a variable for "convex polyhedra", "ordinary polyhedra" (whatever this means), etc. I propose to take these different versions of Euler's theorem as one and the same theorem concerning different variants of the concept of polyhedron. This may be seem only a verbal quibble only, but I think it is not. As we shall see in section 5 , in contrast to the Lakatosian account, the theorems considered as the challenges conceptual variants have to cope with, change in much more dramatic way as Lakatos had been prepared to admit.
} 
of an axiomatic variation of a mathematical concept, or, in Lakatos's terms, as a bold stretching of the number concept.

In the following l'd like to expose the thesis that the dialectical method of axiomatic concept variation may be considered as the fundamental driving force behind the evolution of modern mathematical knowledge. It is the axiomatic method which enables us to vary mathematical concepts in a systematic and effective way. If axiomatic variation of mathematical concepts is the basic mechanism underlying the progress of mathematical knowledge then it is of utmost importance for a philosophical understanding of mathematics to describe this mechanism as precisely as possible.

Obviously, the process of axiomatic variation is not arbitrary. Not anything goes. On the other hand, the process of axiomatic variation is not bound to yield unique results which unanimously are considered as optimal. The evolution of mathematical concepts does not aim at predetermined ends. Rather, axiomatic variation leads to a plurality of variants which compete among each other. Even the leading mathematicians of their time often err in predicting which conceptual variation in the long run will score best. ${ }^{10}$ Most of the conceptual variants which are produced by mathematicians in the course of time are short-lived. They are confined to a rather small temporal interval and do not find fruitful continuation. Most are forgotten, i.e., in evolutionary terms, they die without offspring. Sucessful variants, on the other hand, spread. They are taken up and developed by other authors leading to important new theorems and conceptual connections with other fields of mathematical knowledge. As it seems, many mathematicians are seduced by this fact to occupy a Whiggish perspective which only remembers the winner's approach and is forgetting the other conceptual variants of the past which someday may be revived. ${ }^{11}$

3. Towards a Selection Theory of Mathematical Concepts. If one conceives the history of mathematics as the evolution of mathematical concepts one has to explain what is to be understood by evolution and what are its mechanisms. One should not expect that any existing theory of evolution, say Darwin's or Lamarck's account of biological evolution, or some of their modern successors, is directly applicable to the evolution of mathematical concepts. Rather, the evolution of mathematical concepts may have its own idiosyncratic features

10 For instance, even an expert in the field such as Fubini was rather skeptical whether Lebesgue's new variant of the integral was a useful or not.

11 How dangerous such an attitude may be is nicely described in Rota's "On Reading Collected Papers" (Rota 1997, 248): "In our ahistorical age, it is a fool's paradise to believe that the reading (even the casual scanning) of collected papers is more likely to enrich our knowledge than a feverish plunge into the latest periodicals ... The masters had a variety of ideas that are missing in later acounts of their work." According to Rota, in order to solve an outstanding problem, a mathematician has to seriously study the history of that problem. 
not occurring in any other evolution. For instance, time seems to play a different role in the evolution of mathematical concepts than in, say, the evolution of biological species. To some extent, in mathematics the past is always present. Hence, past forms of mathematical creatures may be revived and come to live again in a way which has no counterpart in biological evolution. The best a general theory of evolution can do is offering a framework or format for a evolutionary understanding of mathematics. Without discussing the pros and cons of other evolutionary accounts I propose an elementary evolutionary theory to be characterized by the following four principles:

(3.1) The Principle of Variation. At any stage in the history of a species, there will be variation among the members of the species. Different variants will have different properties.

(3.2) The Principle of Struggle for Existence. At any stage in the history of a species, more variants come into existence than can survive to reproduce.

(3.3) The Principle of Variation of Fitness. At any stage in the history of a species, some of its variants have characteristics that better dispose them to survive and reproduce.

(3.4) The Principle of Inheritance. Most properties of a variant are inherited by its descendants.

From these principles one may infer that the history of a species will show the modification of that species in the direction of those characteristics which better dispose their bearers to survive and reproduce.

The principles (3.1) - (3.4) may all be traced back to Darwin's evolutionary theory. A theory obeying them, however, is not restricted to the biological realm provided we interpret the key terms such as survival, reproduction and so on in an appropriate non-biological way. Hence, these principles may be considered as a general and abstract scheme for various sorts of evolutionary theories.

If we want to cast the development of mathematical knowledge into the framework of an evolutionary theory the first question we have to answer is what should be considered as the units of selection, i.e., what entities we take as species which evolve in time and have one or more different variants. This is not a trivial question, and it may not have a unique answer. ${ }^{12}$ In this paper, I

${ }^{12}$ Even for biological theories this question has not found an unanimously accepted answer. One may consider, as Darwin did, individuals as the unit of selection. Other fashionable answers are to consider species, or even suborganismal parts as units of selection, i.e., as agents of evolution. 
propose as units of evolution "middle-sized" mathematical concepts, such as function, continuity, differentiability, integral, measure, topology etc. This choice is not the only possible one. For instance, it may be convenient to chose larger conceptual entities such as categories in the sense of Mac Lane and Eilenberg as units of selection. ${ }^{13}$ In the following, however, l'd like to concentrate on a more pedestrian account taking concepts of the above mentioned type as units of selection. The main reason for this procedure is simply that otherwise one had to introduce a lot of conceptual machinery which goes beyond the size of this paper. Actually, I think, categories would even fit better the evolutionary approach presented here.

Although the principles of natural selections have no empirical content the presuppositions of its applicability are empirical. These presuppositions forms the empirical core of evolutionary theory of mathematics. In the next section these general principles are reformulated in order to give them empirical content for the evolution of mathematical concepts.

4. Principles of the Evolution of Mathematical Concepts . In order to get an evolutionary theory of mathematical knowledge started, one has to reformulate the above-mentioned four principles in such a way that they apply to mathematical concepts as units of selection. This means, one has to answer the following questions:

1. What is the "world" in which the evolution takes place?

2. What are the competing units of selection which are the protagonists of the ongoing "struggle of existence" in that world?

In the preceding sections this questions has already been answered as follows: The protagonists of the evolutionary selection process are variants of mathematical concepts. The world in which the competition takes place is a world of proof-problems and conceptual growth in depth and coherence. An elementary example of such an evolution is Lakatos's account of the Euler's conjecture E: For the various variants $p_{i}$ of the polyhedron concept $p$, E defines the crucial task $p_{i}$ has to cope with. A variant $p_{1}$ will supersede a variant $p_{2}$ iff $p_{1}$ gets along better with $\mathrm{E}$ than $\mathrm{p} 2$. Admittedly, the world Lakatos designs for the species of polyhedra concepts is a rather primitive and poorly structured world, and one may well suspect that the worlds mathematical concepts live in, actually are much more complicated. This is indeed the case, as can be seen from

\footnotetext{
${ }^{13}$ Meanwhile, mathematicians are well aware of the selectional character of their research. In particular, this holds for fields such as lattice theory or topology. There, some quite strict "fitness criteria" are generally accepted: for instance, a "good" topological category should possess some nice closure properties, i.e., topological spaces should have products and coproducts, an exponential law for topological spaces should hold etc.
} 
the case of the integral concept. There is not simply one theorematical demand which defines a concept's fitness ("enable a proof of Euler's conjecture") but many.

Before we go into this, let us give a brief sketch of how the four general principles of evolution given in the last section may be applied to the case of the evolution of mathematical concepts.

(4.1) Principle of Variation. At any stage in the history of a mathematics, there will be several different variants of a mathematical concept; these different variants, belonging to the same species, will have different properties but have to cope with the same or at least similar challenges.

Lakatos's case studies in (PR) may be read as evolutionary stories about competing variants of mathematical concepts such as polyedron, continuity, continuum etc. The actual history of mathematics is more complicated. In particular, many more competitors attend the race. This will be discussed in some detail in the next section, is the concept of the integral. I'd like to contend that these examples exhibit the generic case, i.e. they may be considered as species which occur in several different variants differing in "mathematical fitness". Conceptual species which have only a single and unique variant throughout the history of mathematics would be extremely rare, if there are any. ${ }^{14}$

In order to apply the Principle of Struggle for Existence. one has to explain what could it mean that one variant of mathematical concept "survives" and another one "becomes extinct". I propose the following interpretation:

(4.2) The Principle of Competition. At any stage in the history of a mathematics, there is a competition between the variants of concepts. This competition results in a ranking of the competing variants. High ranking for a conceptual variant means that it becomes acknowledged as a useful and fruitful device in proving important theorems and/or giving rise to new and promising theoretical contexts. A variant loses the competition when it falls into oblivion and does not find interesting applications.

\footnotetext{
${ }^{14}$ A candidate for such an exceptional species may be the concept of natural number. It seems hard to find different variants of it. A closer look, however, reveals that even for this apparently undifferentiated species several different variants exist. In axiomatic mathematics, a natural number concept is defined by some version of Peano axioms. Hence, the different variants of the Peano axioms may be considered as defining different conceptual variants of natural numbers. Moreover, if one takes into account that in different toposes different natural number objects exist which may enjoy strikingly different structural properties the principle of variation seems to be secured even for the natural number concept. This basic pluralistic character of mathematical concepts can only be ignored by a narrow elementarist perspective on mathematics which takes into consideration only a small fragment of mathematical knowledge.
} 
For instance, today, Cauchy's variant of the integral may be considered as uninteresting and irrelevant for the further evolution of mathematics. It has been outscored by Riemann's and Lebesgue's extensionally, and moreover, it is to be considered conceptually as a deadlock. Metaphorically, this may be expressed by saying Cauchy's variant is dead. In the course of time, most conceptual variants suffer this destiny. In contrast to biological extinction, however, mathematical concepts have, at least in principle, the chance of being resuscitated. For instance, the Riemann integral has undergone some new conceptual developments and has found new applications (cf. Edalat 1995, Henstock 1991). The Principle of Competition opens the door for sociological considerations. The environment of mathematical concepts, i.e. the theorems which are considered as important and the conceptual relations which are considered as fruithful, is a man-made world. It may depend on changing tastes and preferences. For instance, if the social pressure on the mathematical community is such that only those mathematical achievements are highly esteemed which are directly useful for practical issues the resulting criteria for successful concepts may be quite different from those which reign if other "more theoretical" preferences are made. Thus, in some sense, the evolution of mathematical concepts is relative, it cannot be understood as the unfolding of some absolute conceptual platonic realm. ${ }^{15}$

(4.3) The Principle of Variation of Fitness. The key-term "fitness" of this principle is not easily determined in a non-tautological way (as is the case for its biological counterpart). For mathematical concepts, one, but only one, measure of the degree of fitness is the generality of theorems they allow to prove. A concept for which a more general theorem can be proved is, ceteris paribus, better than one having a more restricted domain of applicability.

Some not overly convincing examples may be found in (PR). A better example is provided by comparing some variants of the concept of the integral to be discussed in the next section. Beyond extensional generality, there are other criteria of fitness such as "fruitfulness" or "conceptual depth". Admittedly, these notions are difficult to nail down. The dependence of an evolutionary account of mathematical knowledge on such a "difficult" notions should be considered, however, as virtue, rendering it a demanding and non-trivial acount. This shows once again, that the standard elementarist accounts do not come even approximately close to the real problems of a philosophy of mathematics.

\section{(4. 4) The Principle of Inheritance. This principle ensures a sort of historical}

15 This opens up the possibility of "alternative mathematics" (cf. Bloor (1991)) Actually, Bloor quite heavily relies on Lakatos' account of mathematical knowledge in order to show that even in mathematical knowledge there may be some sort of "cultural relativity". 
continuity for mathematical concepts. The new variants have to resemble their ancestors in order to meet at least some of the environmental challenges defined by the latter. It is a matter of history of mathematics to elucidate the descendence of the mathematical concepts from their humble beginnings to their sophisticated most recent descendents.

The principle of inheritance ensures some sort of cumulative growth for mathematical knowledge, at least to a certain degree. The cumulative character, however, should not be overestimated. It may well be the case that the modern descendants of mathematical concepts have lost some of the problem-solving capacities of their ancestors, since these problems have become obsolete in modern contexts.

In the next section, some episodes in the evolution of the concept of the integral are sketched according to these evolutionary principles.

5. On the Evolution of the Concept of the Integral. As an example of the evolution of a mathematical concept let us now consider the evolution of the concept of the integral. It goes without saying that only some aspects of the evolution of this concept can be dealt with and even this only in a quite superficial way. ${ }^{16}$

In common usage, one speaks of "the" integral as though this concept were a uniquely determined concept once and for all. This is of course wrong. In highschool mathematics the office of the integral is usually occupied by the Cauchy's variant of the the integral. Most mathematicians probably use to understand by "integral" the Lebesgue's variant of this concept. Specialists in integration theory know a lot other variants, e.g. Denjoy's, Burkill's, Perron's, Taylor's etc. (cf. Henstock 1991). As the recent work of Henstock, Edalat and others exemplifies, the evolution of the concept of the integral can in no way be described as having reached its terminal point (cf. Henstock 1991, Edalat 1997). Rather, a huge and complex family of subspecies has evolved whose members have adapted to a variety of quite different contexts. It goes without saying that I do not intend to describe this complex evolution in its barest outlines. Rather, the rest of this section is devoted to a brief sketch of some aspects of this evolution from Cauchy's variant up to Lebesgue's only.

The relations between the different variants of the concept of the integral is quite intricate. In particular, it cannot be described as a story of linear

\footnotetext{
${ }^{16}$ For a detailed presentation of the origins and the developments of Lebesgue's theory of integration see Hawkins 1970, for the modern post-Lebesgue evolution see Henstock 1991. Counted very roughly the different definition occurring in Hawkins and Henstock several dozens of different variants of the integral have been proposed in the last one hundred and fifty years. This shows that Lakatos's five or six conceptual variants of the polyhedron as exhibited in (PR) form a rather poor collection.
} 
progress. Of course, it is known that the Lebesgue integral is more general than the Riemann integral (to say nothing of Cauchy's variant) in the sense that any on an interval [a,b] Riemann integrable function is Lebesgue integrable but the converse does not hold. For most purposes, Lebesgue's variant turned out to be the most versatile and fruitful one clearly outscoring the more traditional variants. ${ }^{17}$

Let us have a closer look how this happened by starting with Cauchy's variant of the integral. He defined the definite integral of a uniformly continuous ${ }^{18}$ function $f$ as follows: Let $f(x)$ be a uniformly continuous function defined on the closed interval $[a, b]$. Consider a partition of this interval

$$
a=x_{0}<x_{1}<\ldots<x_{n-1}<x_{n}=b \text { and the sum } S(P, f, a, b)=\Sigma\left(x_{i}-x_{i-1}\right)
$$
$f\left(x_{i-1}\right)^{\prime}$

Using the uniform continuity of $f$ he was able to show that for finer and finer partitions the values of $S(P, f, a, b)$ approached a limit. This limit he defined as the definite integral of $f$ on $[a, b]$, to be denoted by $\int(f, a, b)$. Now define $a$ function $F$ by $F(x)=\int(f, a, x)$. For $F$ Cauchy established the following results, known as the Fundamental Theorem of the Calculus:

(5.1) The Fundamental Theorem of Calculus.

$F$ is a primitive function for $f$, i.e., $F^{\prime}=f$.

If $F$ is a primitive function for $f$ all primitive functions for $f$ are of

the form $G=F+c$, where $c$ is a constant. That is, if $G$ is a function with a continuous derivative $G^{\prime}$, then $\int\left(G^{\prime}, a, x\right)=G(x)$ $\mathrm{G}(\mathrm{a})$.

If $G$ is a function such that $G^{\prime}(x)=0$ for all $x$ in $[a, b]$ then

$\mathrm{G}(\mathrm{x})=\mathrm{c}$ for some constant $\mathrm{c}$.

Since the times of Leibniz and Newton, any viable variant of the integral concept had to satisfy some version of the Fundamental Theorem. In the course of time, especially in the 19th century, however, the authority of the Fundamental Theorem as the crucial prove experiment for an admissible variant

\footnotetext{
17 This outcome wasn't that clear in the first quarter of the century (cf. Hahn 1914, 1915). Several attempts were made to modify Riemann's definition of the integral so that it would accommodate unbounded integrands and encompass conditionally convergent integrals. It took some time for that Lebesgue's account changed the terms of competition in such a way that the superiority of Lebesgue's variant was generally acknowledged, at least for some time and some domain of applications.

18 Of course, the concept of a uniformly continuous function was explicitly defined only after Cauchy. However, Cauchy often implicitly assumed a function to be uniformly continuous when he allegedly dealt with continuous functions.
} 
of the integral concept diminuished. ${ }^{19}$ Other challenges for the integral concept came to the fore, for instance, various types of convergence theorems which described the successful interplay of integral and various limit operations. One reason for this changing of environmental challenges was the gradual expansion of the domain of functions for which the Fundamental Theorem was assumed to hold.

Fulfilling the demands of the Fundamental Theorem became more and more difficult when the domain of functions for which the Fundamental Theorem was designed to be applicable got more and more expanded. In other words, the features of the environment of the concept of the integral gradually changed. As is well known, one of the main protagonists of the change was Dirichlet who for the first time introduced the concept of a truly "general" function to be conceived of as a general rule correlating the elements of the domain and the range of the function. Dirichlet was the first mathematician to call attention to the existence of functions that are discontinuous on an infinite set of points in a finite interval and to the problem of extending the concept of the integral to functions of this nature. For some time, the best response to this new challenge was Riemann's variant of the integral: He interpreted the meaning of integrability in terms of the Cauchy sums of the function $f(x)$ to be integrated: Under what conditions on the function $f(x)$ do these sums approach a unique limit as the norm of the corresponding partitions approaches zero? In order to answer this question Riemann formulated two equivalent conditions. Then he showed that his definition was extensionally superior to Cauchy's (and Dirichlet's) by constructing functions which were discontinuous on a dense set of real numbers but nevertheless integrable according to his integral but not in the sense of Dirichlet and Cauchy. As is pointed by Hawkins $(1970,18)$, the advantage of Riemann's variant of the integral is not exhausted by its larger domain of applicability. In hindsight, one may see in Riemann's integrability conditions the germs of the concepts of Jordan measurability and outer content which were bound to play a crucial role in modern theory of integration as it was to be developed much later by Lebesgue. Conceptual progress cannot be characterized by extensional considerations alone. If the competition among the various integral concepts could be fully described in this extensional way it would essentially follow the lines of Lakatos's discussion of the conceptstretching of the concept of the polyhedron carried out in (PR) (cf. also Fine 1981). Actually, things are much more complex. Conceptual mathematical progress is not measured exclusively by the expansion of the domain of

19 For instance, discoveries of Dini, Volterra and others show that the second part of Fundamental Theorem (5.1(2)) is not generally valid for Riemann's variant of the integral, since there are functions with bounded non-integrable derivatives. Lebesgue's variant in some sende restores this part of the Fundamental Theorem, at lest for bounded derivatives. On the other hand, for Lebesgue's variant, the first part of the Fundamental Theorem (5.1)(1) does not hold in full generality. It only holds in a version "almost everywhere" (cf. Hawkins 1970, 137). 
intended application Rather, the development of new conceptual relations plays an essential role. The superiority of a new conceptual variant is also, and one may even say, mainly, demonstrated by the new lines of research and the new perspectives it opens. Thus, the evolutionary value of a new variant of a mathematical concept is assessed also by the new conceptual relations it opens. In the case of the evolution of the integral this is most impressively demonstrated by Lebesgue's integral. As is well-known, Lebesgue's variant is extensionally superior to Riemann's. This advantage, however, is not the most important one. Lebesgue's new variant of the integral turned out to be a real mutation, so to speak. It opened up a completely new field and defined a new framework for the concept of the integral. For the first time, it elucidated the intimate relation between the concepts of integral, measure, and topology.

From Lebesgue (and Borel) onwards any variant of the concept of the integral had to consider relations with the conceptual field of measure theory and topology which were quite alien to Cauchy's concept of the integral and only dimly visible for Riemannan's variant. Hence, the transition from the Riemann variant to the Lebesgue variant cannot be fully understood as simply an extensional expansion. Rather, it also involves an intensional, i.e. conceptual progress insofar as the Lebesgue variant is able to build up connections and interrelations to many more other conceptual fields. ${ }^{20}$

6. Concluding Remarks. As is shown by the ongoing evolution of mathematical knowledge the "essence" of mathematics is not to be found in some hidden nature of mathematical objects, rather it is revealed in the specifics of the ongoing evolutionary process of mathematical knowledge, or, as the Neokantians such as Cohen and Natorp would have formulated it some one hundred years ago: the fact of mathematics (science) is a fact of becoming ("Werdefaktum"). ${ }^{21}$ If this is true, as I think it is, it is of the utmost importance for a philosophical understanding of mathematics to get some insights into the mechanisms of this ongoing process. The best device for an insightful description of this process are provided by some sort of evolutionary theory.

\footnotetext{
20 This points to the fact that the evolution of mathematical concepts should be thought of as the evolution of a single concept but rather as a group evolution of many concepts simultaneously.

21 See Cohen's Logik der reinen Erkenntnis (Cohen 1977(1902). The process character of scientific knowledge is even starker emphasized in Natorp's Die logischen Grundlagen der exakten Wissenschaften (Leipzig 1910). There one finds in the forth section the following radical thesis: "The fact of science must be understood as a "fieri" (becoming). The "fieri" alone is the fact: all being which science attempts to fix, has to be resolved in the great current of becoming. Only this becoming can finally be said to be." This paper is, of course, not the appropriate place to deal with the Neo-Kantian philosophy of science and mathematics in any greater depth. l'd like to attention to the fact that the Neo-Kantian approach emphasized the conceptual unity between science and mathematics which has largely gone unnoticed in the traditional positvist philosophy of science.
} 
In order to apply such a theory a necessary prerequisite is to understand that mathematical concepts are not eternal unalterable "Platonic" entities. Rather, they use to occur in several different variants which develop in history. These variants differ in fitness concerning the wealth and value of the theorems which can be proved for them. Variation may occur on different levels, for instance, on the level of concepts, on the level of propositions, on the level of theories. This ubiquity of variation renders modern mathematical knowledge a definitely un-Platonic epistemic endeavor rendering variation (on which level whatsoever) as the very essence of mathematics. Variation is not a 'noise' which may be ignored or filtered out by clever philosophical devices in order to grasp the "essence" of mathematics. I consider it as the great achievement of Lakatos's philosophy of mathematics to have paved the way for this insight.

The acceptance of general framework for a philosophical understanding of mathematics may not be too instructive. The evolution of mathematical knowledge exhibits some very special features which it does not share with other evolutions. Hence, it is of crucial importance to explicate these special features:

(1) The environment in which the evolution takes places, may change in a rather quick and drastic manner. This change may be brought about, at least partially, by the conceptual innovations themselves.

(2) Temporarily unsuccessful variants may survive in a "frozen state" and may be resuscitated. ${ }^{22}$ Hence, compared with other evolutions the struggle of existence in the conceptual evolution of mathematics is less cruel. Although there is a strong competition among conceptual variants this need not lead to the total extinction of the inferior variants. They may survive in some niche or, perhaps, may be resuscitated after the terms of competition have changed. ${ }^{23}$

(3) Conceptual evolution in mathematics is a group affair. That is to say, not a single concept undergoes, rather a group of connected concepts evolves simultaneously. For instance, the evolution of the concepts of the integral is intimately related to the evolution of the concept of measure.

These features show that an evolutionary conceptualization cannot rely on some stories told about evolutions that took place elsewhere. Rather, philosophers and historians of mathematics have yet to invent the language for

\footnotetext{
${ }^{22}$ Cf.Footnote 15.

${ }^{23}$ Lakatos has taken into consideration this fact later in his account "Methodology of Scientific Research Programmes".
} 
telling evolutionary stories in the case of mathematics. The first steps for this endeavour have been done by Lakatos and his theory of concept formation and concept stretching.

\section{References:}

Bloor, D., 1991 (1976), Knowledge and Social Imagery, Chicago, The University of Chicago Press.

Brandon, R.N., 1996, Concepts and Methods in Evolutionary Biology, Cambridge, Cambridge University Press.

Cohen, H., 1902(1977), Die Logik der reinen Erkenntnis (Hermann Cohen Werke 6), Hildesheim und New York, Olms.

Corfield, D., 1997, Assaying Lakatos's Philosophy of Mathematics, STUDIES IN HISTORY AND PHILOSOPHY OF SCIENCE 28, 99 - 121.

Edalat, A., 1997, Domains for Computation in Mathematics, Physics and Exact Real Arithmetics, THE BULLETIN OF SYMBOLIC LOGIC 3, 401 - 452

Elstrodt, J., 1996, Maß- und Integrationstheorie, Berlin, Springer.

Ernest, P., 1997, The Legacy of Lakatos: Reconceptualizing the Philosophy of Mathematics, PHILOSOPHIA MATHEMATICA 5, 116 - 134.

Fine, A., 1981, Conceptual Change in Mathematics and Science: Lakatos' Stretching Refined, Proceedings of the 1978 Biennial Meeting of the Philosophy of of Science Association (PSA), vol. 2, 328 - 341.

Hamilton, W.R., 1967, The Mathematical Papers of Sir William Rowan Hamilton, vol. 3, Algebra, Cambridge, Cambridge University Press.

Hahn, Hans, 1997(1914), Über Annäherung an Lebesguesche Integrale durch Riemannsche Summen, in Gesammelte Abhandlungen 3, herausgegeben von L. Schmetterer und K. Sigmund, Wien, Springer, 17 - 48.

Hahn, Hans, 1997(1915), Über eine Verallgemeinerung der Riemannschen Integralfunktion, in Gesammelte Abhandlungen 3, herausgegeben von L. Schmetterer und K. Sigmund, Wien, Springer, $49-64$.

Hawkins, Th., 1970, Lebesgue's Theory of Integration, Its Origins and Developments, Madison, The University of Wisconsin Press.

Henstock, R., 1991, The General Theory of Integration, Oxford, Clarendon Press.

Lakatos, I., 1976, Proofs and Refutations: The Logic of Mathematical Discovery, Cambridge, Cambridge University Press.

Lakatos, I., 1978, Philosophical Papers. Volumes 1 and 2, Cambridge, Cambridge University Press.

Larvor, B.P., 1997, Lakatos as Historian of Mathematics, PHILOSOPHIA MATHEMATICA (3) 5, 42 - 64. 
Koetsier, T., 1991, Lakatos' Philosophy of Mathematics. A Historical Approach, Amsterdam, North-Holland.

Natorp, P., 1910, Die logischen Grundlagen der exakten Wissenschaften, Leipzig und Berlin. Teubner.

Pickering, A., 1995, The Mangle of Practice, Time, Agency, and Science, Chicago, The University of Chicago Press.

Rota, G.C., 1997, Indiscrete Thoughts, edited by F. Palombi, Boston, Birkhäuser.

Toulmin, St., 1972, Human Understanding I. General Introduction and Part I: The Collective Use and Evolution of Concepts, Princeton, Princeton University Press. 